Research Article

\title{
Performance Analysis in DF Energy Harvesting Full-Duplex Relaying Network with MRC and SC at the Receiver under Impact of Eavesdropper
}

\author{
Phu Tran Tin $\mathbb{D}^{1},{ }^{1}$ Van-Duc Phan $\mathbb{D}^{2},{ }^{2}$ Dong Si Thien Chau $\mathbb{D}^{3},{ }^{3}$ Tan N. Nguyen $\mathbb{D}^{4}{ }^{4}$ \\ and Phu X. Nguyen (D) ${ }^{5}$ \\ ${ }^{1}$ Faculty of Electronics Technology, Industrial University of Ho Chi Minh City, Ho Chi Minh City, Vietnam \\ ${ }^{2}$ Faculty of Automobile Technology, Van Lang University, Ho Chi Minh City, Vietnam \\ ${ }^{3}$ Modeling Evolutionary Algorithms Simulation and Artificial Intelligence, Faculty of Electrical \& Electronics Engineering, \\ Ton Duc Thang University, Ho Chi Minh City, Vietnam \\ ${ }^{4}$ Wireless Communications Research Group, Faculty of Electrical and Electronics Engineering, Ton Duc Thang University, \\ Ho Chi Minh City, Vietnam \\ ${ }^{5}$ Department of Computer Fundamentals, FPT University, Ho Chi Minh City, Vietnam
}

Correspondence should be addressed to Dong Si Thien Chau; dongsithienchau@tdtu.edu.vn

Received 12 January 2021; Revised 2 April 2021; Accepted 19 June 2021; Published 28 June 2021

Academic Editor: Jit S. Mandeep

Copyright () 2021 Phu Tran Tin et al. This is an open access article distributed under the Creative Commons Attribution License, which permits unrestricted use, distribution, and reproduction in any medium, provided the original work is properly cited.

This paper investigates the decode-and-forward (DF) full-duplex (FD) relaying system under the presence of an eavesdropper. Moreover, the relay node is able to harvest energy from a transmitter, and then it uses the harvested energy for conveying information to the receiver. Besides, both two-hop and direct relaying links are taking into consideration. In the mathematical analysis, we derived the exact expressions for intercept probability and outage probability (OP) by applying maximal ratio combining (MRC) and selection combining (SC) techniques at the receiver. Next, the Monte Carlo simulation is performed to validate the mathematical analysis. The results show that the simulation curves match the mathematic expressions, which confirms the analysis section.

\section{Introduction}

Radio frequency- (RF-) enabled wireless power transfer (WPT) has recently become a promising technique to overcome the energy limitation for wireless communication networks [1-10]. Moreover, because RF signal is able to carry both energy and information, one attractive direction is to transmit information and energy simultaneously (SWIPT) jointly. The SWIPT relaying networks have been intensively studied in [11-16]. Besides energy harvesting, physical layer security for relay networks received great attention from researchers [17-22]. Recent advances in self-interference cancellation (SIC) techniques achieve high SI reduction. Therefore, FD relaying communications become a promising solution to overcome the spectrum scarcity of wireless systems [23-25].

In this paper, we consider a new system model to investigate the trade-off between physical layer security (PLS) and reliability for an energy-constrained FD relaying network. Further, the direct link between transmitter and receiver is taken into account to improve the total network performance. Moreover, the maximal ratio combining (MRC) and selection combining (SC) protocols are exploited at the eavesdropper and destination to enhance their received rate. The research contributions are summarized as follows: 
(i) We present an FD- and SWIPT-assisted relaying network in decode-and-forward (DF) under the presence of a direct link. Particularly, an eavesdropper is able to overhear the information transmission from source to destination via a relay. Moreover, an FDenabled relay node is able to get energy from a transmitter and use it to transfer signals to the a receiver. Notably, the relay node can simultaneously receive information from the source and transmit it to the destination using the FD technique.

(ii) We derive closed-form expressions of intercept probability (IP) at the eavesdropper E and outage probability (OP) at the destination $\mathrm{D}$ in maximal ratio combining (MRC) and selection combining (SC) techniques.

(iii) The correctness of the developed analysis is validated through the Monte Carlo simulation. On one hand, we investigate the security perspective in terms of intercept probability. On the other hand, system reliability is also studied through outage probability. Consequently, a trade-off between IP and OP can provide many insightful and useful perspectives for system designers.

\section{System Model}

In Figure 1, we consider a relaying network where a relay $\mathrm{R}$ aids in conveying data from a transmitter $S$ to a receiver $D$ in the presence of one eavesdropper E. In particular, an eavesdropper is trying to get the information from $\mathrm{S}$ and $\mathrm{R}$ by applying maximal ratio combining (MRC) and selection combining (SC) techniques. In Figure 2, the relay $\mathrm{R}$ can harvest energy from the source during $\alpha T$. In the remaining time, $(1-\alpha) T$, the information process is executed.

We assume that the channel between two users follows block Rayleigh fading, where channel coefficients are unchanged during a time frame and change independently across time frames. Moreover, let us denote $h_{\mathrm{XY}}$ for $\mathrm{XY} \in\{\mathrm{SR}, \mathrm{RD}, \mathrm{RR}, \mathrm{SD}, \mathrm{RE}, \mathrm{SE}\}$ as the channel coefficient of the link between nodes $X$ and $Y$. Because the channels are Rayleigh distribution, the channel gains such as $\left|h_{\mathrm{RD}}\right|^{2}$ and $\left|h_{\mathrm{SD}}\right|^{2}$ are exponential random variables (RVs) whose cumulative distribution function (CDF) and probability density function (PDF) are, respectively, represented as

$$
\begin{aligned}
& F_{X}(x)=1-\exp (-\lambda x), \\
& f_{X}(x)=\frac{\partial F_{X}(x)}{\partial x}=\lambda \exp (-\lambda x),
\end{aligned}
$$

where $\lambda$ is rate parameter of exponential distribution.

The received signal at the relay can be expressed as

$$
y_{R}=h_{\mathrm{SR}} x_{S}+h_{\mathrm{RR}} x_{R}+n_{R}
$$

where $x_{S}$ is the energy symbol and $E\left\{\left|x_{S}\right|^{2}\right\}=P_{S}, x_{R}$ is the loopback interference due to full-duplex relaying and satisfies $E\left\{\left|x_{R}\right|^{2}\right\}=P_{R}$, where $E\{\cdot\}$ denotes the expectation operation. $n_{R}$ denotes the zero mean additive white Gaussian noise $(A W G N)$ with variance $\mathrm{N}_{0}$.

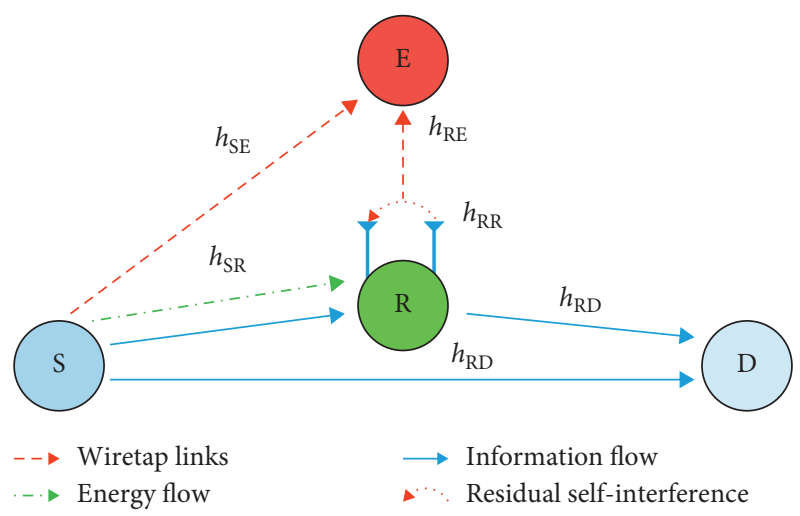

Figure 1: System model.

$\mathrm{T}$

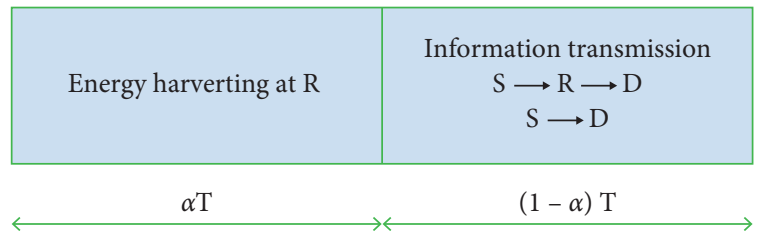

Figure 2: IT and EH processes.

At the first phase, the harvested energy at the relay can be computed by

$$
E_{R}=\eta \alpha \mathrm{TP}_{S}\left|h_{\mathrm{SR}}\right|^{2}
$$

where $0<\eta \leq 1$ denotes the energy conversion efficiency.

From (3), the average transmit power of the relay node can be obtained as

$$
P_{\mathrm{R}}=\frac{E_{\mathrm{R}}}{(1-\alpha) T}=\frac{\eta \alpha P_{\mathrm{S}}\left|h_{\mathrm{SR}}\right|^{2}}{(1-\alpha)}=\kappa P_{\mathrm{S}}\left|h_{\mathrm{SR}}\right|^{2},
$$

where $\kappa=\eta \alpha / 1-\alpha$.

Next, in the second phase, the eavesdropper $\mathrm{E}$ may intercept signals from both relay $\mathrm{R}$ and source S. Nevertheless, source $S$ also generates artificial noise $\tilde{x}_{S}$ to prevent $\mathrm{E}$ from overhearing the source information. Moreover, since the relay $\mathrm{R}$ and destination $\mathrm{D}$ are legitimate users, they are assumed to know the artificial noise created by S. Consequently, they can cancel the artificial noise at the receiver circuit. Therefore, the received signal at $\mathrm{E}$ from relay $\mathrm{R}$ and source $\mathrm{S}$ can be, respectively, expressed as

$$
y_{E}^{I}=h_{\mathrm{RE}} x_{R}+n_{E}^{I}, y_{E}^{\mathrm{II}}=h_{\mathrm{SE}} x_{S}+h_{\mathrm{SE}} \tilde{x}_{S}+n_{E}^{\mathrm{II}},
$$

where $n_{\mathrm{E}}={ }_{n}^{\mathrm{EI}}=n_{\mathrm{E}}^{\mathrm{II}}$ is the AWGN with variance $N_{0}$.

Since we adopt the decode-and-forward (DF) protocol, the signal to interference noise ratios (SINR) at the eavesdropper in the second phase from (5) are, respectively, given by

$$
\gamma_{E}^{I}=\frac{\left|h_{\mathrm{RE}}\right|^{2} P_{R}}{N_{0}}=\kappa \Psi\left|h_{\mathrm{SR}}\right|^{2}\left|h_{\mathrm{RE}}\right|^{2}, \gamma_{E}^{I I}=\frac{\Psi\left|h_{\mathrm{SE}}\right|^{2}}{\Psi\left|h_{\mathrm{SE}}\right|^{2}+1},
$$

where $\Psi=P_{s} / N_{0}$. 
As mentioned in the above discussion, the destination $\mathrm{D}$ can cancel the artificial noise from source S. Consequently, the received signal at the destination from relay $\mathrm{R}$ and source $\mathrm{S}$ during the second phase can be expressed as

$$
y_{\mathrm{D}}^{\mathrm{I}}=h_{\mathrm{RD}} x_{\mathrm{R}}+n_{\mathrm{D}}^{\mathrm{I}}, y_{\mathrm{D}}^{\mathrm{II}}=h_{\mathrm{SD}} x_{\mathrm{S}}+n_{\mathrm{D}}^{\mathrm{II}} \text {, }
$$

where $n_{D}=n_{D}^{\mathrm{I}}=n_{D}^{\mathrm{II}}$ is the AWGN with variance $\mathrm{N}_{0}$.

\section{Intercept Probability (IP) Analysis}

Destination $\mathrm{D}$ will be intercepted if $\mathrm{E}$ can successfully wiretap signal; that is, $\gamma_{E} \geq \gamma_{\mathrm{th}}$, where $\gamma_{\text {th }}=2^{\mathrm{R}}-1$ and $\mathrm{R}$ is the target rate.

Therefore, the IP of the system can be expressed as

$$
\mathrm{IP}=\operatorname{Pr}\left(\gamma_{E} \geq \gamma_{t h}\right) \text {. }
$$

3.1. Instantaneous End-to-End SNR at E Using the MRC Technique. In this case, the end-to-end SNR at E from (6) can be given by

$$
\gamma_{E}^{\mathrm{MRC}}=\gamma_{E}^{\mathrm{I}}+\gamma_{E}^{\mathrm{II}}=\kappa \Psi\left|h_{\mathrm{SR}}\right|^{2}\left|h_{\mathrm{RE}}\right|^{2}+\frac{\Psi\left|h_{\mathrm{SE}}\right|^{2}}{\Psi\left|h_{\mathrm{SE}}\right|^{2}+1} .
$$

Then, the IP in (7) can be rewritten as

$$
\begin{aligned}
\mathrm{IP}_{\mathrm{MRC}} & =\operatorname{Pr}\left(\kappa \Psi\left|h_{\mathrm{SR}}\right|^{2}\left|h_{\mathrm{RE}}\right|^{2}+\frac{\Psi\left|h_{\mathrm{SE}}\right|^{2}}{\Psi\left|h_{\mathrm{SE}}\right|^{2}+1} \geq \gamma_{\text {th }}\right) \\
& =\operatorname{Pr}\left(X+Y \geq \gamma_{\mathrm{th}}\right)=1-\int_{0}^{\gamma_{\mathrm{th}}} F_{X}\left(\gamma_{\mathrm{th}}-y\right) \times f_{Y}(y) d y,
\end{aligned}
$$

where $X=\kappa \Psi\left|h_{\mathrm{SR}}\right|^{2}\left|h_{\mathrm{RE}}\right|^{2}, Y=\Psi\left|h_{\mathrm{SE}}\right|^{2} / \Psi\left|h_{\mathrm{SE}}\right|^{2}+1$.

In order to find the probability in (10), we have to find the CDF of $X$ and PDF of $Y$. So, the CDF of $X$ can be calculated by

$$
\begin{aligned}
F_{X}(x) & =\operatorname{Pr}(X<x)=\operatorname{Pr}\left(\kappa \Psi\left|h_{\mathrm{SR}}\right|^{2}\left|h_{\mathrm{RE}}\right|^{2}<x\right) \\
& =\operatorname{Pr}\left(\left|h_{\mathrm{SR}}\right|^{2}<\frac{x}{\kappa \Psi\left|h_{\mathrm{RE}}\right|^{2}}\right)=\int_{0}^{\infty} F_{\left|h_{\mathrm{SR}}\right|^{2}}\left(\left.\frac{x}{\kappa \Psi y}|| h_{R E}\right|^{2}=y\right) \times f_{\left|h_{R E}\right|^{2}}(y) d y \\
& =1-\int_{0}^{\infty} \lambda_{\mathrm{RE}} \exp \left(-\frac{\lambda_{\mathrm{SR}} x}{\kappa \Psi y}-\lambda_{\mathrm{RE}} y\right) d y .
\end{aligned}
$$

By applying (Eq. 3.324.1, [26]), (11) can be obtained by

Next, the CDF of Y can be formulated as

$$
F_{X}(x)=1-2 \sqrt{\frac{\lambda_{\mathrm{SR}} \lambda_{\mathrm{RE}} x}{\kappa \Psi}} \times K_{1}\left(2 \sqrt{\frac{\lambda_{\mathrm{SR}} \lambda_{\mathrm{RE}} x}{\kappa \Psi}}\right) .
$$

$$
\begin{aligned}
F_{Y}(y) & =\operatorname{Pr}(Y<y)=\operatorname{Pr}\left(\frac{\Psi\left|h_{\mathrm{SE}}\right|^{2}}{\Psi\left|h_{\mathrm{SE}}\right|^{2}+1}<y\right) \\
& =\operatorname{Pr}\left(\left|h_{\mathrm{SE}}\right|^{2}<\frac{y}{\Psi(1-y)}\right)=1-\exp \left(-\frac{\lambda_{\mathrm{SE}} y}{\Psi(1-y)}\right) \text {, with } 0<y<1 .
\end{aligned}
$$

The PDF of $\mathrm{Y}$ is given by

$$
f_{Y}(y)=\frac{\partial F_{y}(y)}{\partial y}=\frac{\lambda_{\mathrm{SE}}}{\Psi} \times \frac{\exp \left(-\lambda_{\mathrm{SE}} y / \Psi(1-y)\right)}{(1-y)^{2}} \text {. }
$$

Applying (12) and (14), the IP, in this case, can be claimed by

$$
\begin{aligned}
& I P_{\mathrm{MRC}}=1-\int_{0}^{1}\left\{1-2 \sqrt{\frac{\lambda_{\mathrm{SR}} \lambda_{\mathrm{RE}}\left(\gamma_{\mathrm{th}}-y\right)}{\kappa \Psi}} \times K_{1}\left(2 \sqrt{\frac{\lambda_{\mathrm{SR}} \lambda_{\mathrm{RE}}\left(\gamma_{\mathrm{th}}-y\right)}{\kappa \Psi}}\right)\right\} \\
& \times \frac{\lambda_{\mathrm{SE}}}{\Psi} \times \frac{\exp \left(-\lambda_{\mathrm{SE}} y / \Psi(1-y)\right)}{(1-y)^{2}} d y .
\end{aligned}
$$


3.2. Instantaneous End-to-End SNR at E Using the SC Technique. In this case, the end-to-end SNR at E can be given by

$$
\begin{aligned}
\gamma_{E}^{\mathrm{SC}} & =\max \left(\gamma_{E}^{\mathrm{I}}, \gamma_{E}^{\mathrm{II}}\right)=\max \left(\kappa \Psi\left|h_{\mathrm{SR}}\right|^{2}\left|h_{\mathrm{RE}}\right|^{2}, \frac{\Psi\left|h_{\mathrm{SE}}\right|^{2}}{\Psi\left|h_{\mathrm{SE}}\right|^{2}+1}\right) \\
& =\max (X, Y) .
\end{aligned}
$$

Then, the IP can be expressed as

$$
\begin{aligned}
\mathrm{IP}_{\mathrm{SC}} & =\operatorname{Pr}\left(\gamma_{E}^{\mathrm{SC}} \geq \gamma_{\mathrm{th}}\right)=\operatorname{Pr}\left(\max (X, Y) \geq \gamma_{\mathrm{th}}\right) \\
& =1-\operatorname{Pr}\left(X<\gamma_{\mathrm{th}}\right) \operatorname{Pr}\left(Y<\gamma_{\text {th }}\right) .
\end{aligned}
$$

By applying (12) and (13), the expression of $\mathrm{IP}_{\mathrm{SC}}$ can be expressed as

$$
\mathrm{IP}_{\mathrm{SC}}=1-\left\{1-\exp \left(-\frac{\lambda_{\mathrm{SE}} \gamma_{\mathrm{th}}}{\Psi\left(1-\gamma_{\mathrm{th}}\right)}\right)\right\} \times\left\{1-2 \sqrt{\frac{\lambda_{\mathrm{SR}} \lambda_{\mathrm{RE}} \gamma_{\mathrm{th}}}{\kappa \Psi}} \times K_{1}\left(2 \sqrt{\frac{\lambda_{\mathrm{SR}} \lambda_{\mathrm{RE}} \gamma_{\mathrm{th}}}{\kappa \Psi}}\right)\right\}
$$

\section{Outage Probability (OP) Analysis}

The OP can be defined by

$$
\mathrm{OP}=\operatorname{Pr}\left(\gamma_{D}<\gamma_{\mathrm{th}}\right) \text {. }
$$

4.1. Instantaneous End-to-End SNR at D Using the MRC Technique. From (2) and (7), outage probability of relay link can be computed at

$$
\begin{array}{r}
\mathrm{OP}_{1}=\operatorname{Pr}(\text { source }- \text { relay or relay }- \text { destination is in outage }) \\
=\operatorname{Pr}\left(\min \left(\frac{1}{\kappa\left|h_{\mathrm{RR}}\right|^{2}}, \kappa \Psi\left|h_{\mathrm{SR}}\right|^{2}\left|h_{\mathrm{RD}}\right|^{2}\right)<\gamma_{\text {th }}\right) .
\end{array}
$$

From (20), we can see that, to successfully receive data at the destination $\mathrm{D}$, the system needs to decode in the first and second hop.

Equivalently, we can represent the end-to-end SINR of the relay path by

$$
T=\min \left(\frac{1}{\kappa\left|h_{\mathrm{RR}}\right|^{2}}, \kappa \Psi\left|h_{\mathrm{SR}}\right|^{2}\left|h_{\mathrm{RD}}\right|^{2}\right) .
$$

By using MRC technique, the received SINR at destination can be given as

$$
\gamma_{\mathrm{D}}^{\mathrm{MRC}}=\min \left(\frac{1}{\kappa\left|h_{\mathrm{RR}}\right|^{2}}, \kappa \Psi\left|h_{\mathrm{SR}}\right|^{2}\left|h_{\mathrm{RD}}\right|^{2}\right)+\Psi\left|h_{\mathrm{SD}}\right|^{2}=T+Z,
$$

where $Z=\Psi\left|h_{\mathrm{SD}}\right|^{2}$.

The OP, in this case, can be expressed by

$$
\mathrm{OP}_{\mathrm{MRC}}=\operatorname{Pr}\left(T+Z<\gamma_{\mathrm{th}}\right)=\int_{0}^{\infty} F_{T}\left(\gamma_{\mathrm{th}}-z\right) \times f_{Z}(z) \mathrm{dz}
$$

From $(21), F_{T}(t)$ can be calculated as

$$
\begin{aligned}
F_{T}(t) & =\operatorname{Pr}(T<t)=\operatorname{Pr}\left(\min \left(\frac{1}{\kappa\left|h_{\mathrm{RR}}\right|^{2}}, \kappa \Psi\left|h_{\mathrm{SR}}\right|^{2}\left|h_{\mathrm{RD}}\right|^{2}\right)<t\right) \\
& =1-\underbrace{\operatorname{Pr}\left(1 / \kappa\left|h_{\mathrm{RR}}\right|^{2} \geq t\right)}_{P_{1}} \underbrace{\operatorname{Pr}\left(\kappa \Psi\left|h_{\mathrm{SR}}\right|^{2}\left|h_{\mathrm{RD}}\right|^{2} \geq t\right)}_{P_{2}},
\end{aligned}
$$

where

$$
\begin{aligned}
& P_{1}=\operatorname{Pr}\left(\frac{1}{\kappa\left|h_{\mathrm{RR}}\right|^{2}} \geq t\right)=\operatorname{Pr}\left(\left|h_{\mathrm{RR}}\right|^{2} \leq \frac{1}{\kappa t}\right)=1-\exp \left(-\frac{\lambda_{\mathrm{RR}}}{\kappa t}\right), \\
& P_{2}=1-\operatorname{Pr}\left(\kappa \Psi\left|h_{\mathrm{SR}}\right|^{2}\left|h_{\mathrm{RD}}\right|^{2}<t\right)=2 \sqrt{\frac{\lambda_{\mathrm{SR}} \lambda_{\mathrm{RD}} t}{\kappa \Psi}} \times K_{1}\left(2 \sqrt{\frac{\lambda_{\mathrm{SR}} \lambda_{\mathrm{RD}} t}{\kappa \Psi}}\right) .
\end{aligned}
$$

Substituting (23) and (24) into (22), $F_{T}(t)$ can be mathematically calculated as

$$
\begin{aligned}
F_{T}(t)= & 1-2\left\{1-\exp \left(-\frac{\lambda_{\mathrm{RR}}}{\kappa t}\right)\right\} \times \sqrt{\frac{\lambda_{\mathrm{SR}} \lambda_{\mathrm{RD}} t}{\kappa \Psi}} \\
& \times K_{1}\left(2 \sqrt{\frac{\lambda_{\mathrm{SR}} \lambda_{\mathrm{RD}} t}{\kappa \Psi}}\right) .
\end{aligned}
$$

By substituting (25) into (21), $\mathrm{OP}_{\mathrm{MRC}}$ can be given by 


$$
\mathrm{OP}_{\mathrm{MRC}}=\int_{0}^{\infty}\left\{\begin{array}{c}
1-2\left\{1-\exp \left(-\frac{\lambda_{\mathrm{RR}}}{\kappa\left(\gamma_{\mathrm{th}}-z\right)}\right)\right\} \\
\times \sqrt{\frac{\lambda_{\mathrm{SR}} \lambda_{\mathrm{RD}}\left(\gamma_{\mathrm{th}}-z\right)}{\kappa \Psi}} \times K_{1}\left(2 \sqrt{\frac{\lambda_{\mathrm{SR}} \lambda_{\mathrm{RD}}\left(\gamma_{\mathrm{th}}-z\right)}{\kappa \Psi}}\right)
\end{array}\right\} \times \frac{\lambda_{\mathrm{SD}}}{\Psi} \exp \left(-\frac{\lambda_{\mathrm{SD}} z}{\Psi}\right) \mathrm{d} z
$$

4.2. Instantaneous End-to-End $S N R$ at D Using the $S C$ Hence, the OP can be calculated as Technique. Similar to MRC technique as mentioned above, the overall SNR at D can be given by

$$
\gamma_{D}^{\mathrm{SC}}=\max \left\{\min \left(\frac{1}{\kappa\left|h_{\mathrm{RR}}\right|^{2}}, \kappa \Psi\left|h_{\mathrm{SR}}\right|^{2}\left|h_{\mathrm{RD}}\right|^{2}\right), \Psi\left|h_{\mathrm{SD}}\right|^{2}\right\} .
$$

$$
\begin{aligned}
\mathrm{OP}_{\mathrm{SC}} & =\operatorname{Pr}\left(\gamma_{D}^{\mathrm{SC}}<\gamma_{\mathrm{th}}\right)=\operatorname{Pr}\left[\max \left\{\min \left(\frac{1}{\kappa\left|h_{\mathrm{RR}}\right|^{2}}, \kappa \Psi\left|h_{\mathrm{SR}}\right|^{2}\left|h_{\mathrm{RD}}\right|^{2}\right), \Psi\left|h_{\mathrm{SD}}\right|^{2}\right\}<\gamma_{\mathrm{th}}\right] \\
& =\underbrace{\operatorname{Pr}\left(\Psi\left|h_{\mathrm{SD}}\right|^{2}<\gamma_{\mathrm{th}}\right)}_{P_{3}\left[\min \left(\frac{1}{\kappa\left|h_{\mathrm{RR}}\right|^{2}} \kappa \Psi\left|h_{\mathrm{SR}}\right|^{2}\left|h_{\mathrm{RD}}\right|^{2}\right)<\gamma_{\mathrm{th}}\right]},
\end{aligned}
$$

where

$$
\begin{aligned}
P_{3} & =\operatorname{Pr}\left[\min \left(\frac{1}{\kappa\left|h_{\mathrm{RR}}\right|^{2}}, \kappa \Psi\left|h_{\mathrm{SR}}\right|^{2}\left|h_{\mathrm{RD}}\right|^{2}\right)<\gamma_{\mathrm{th}}\right] \\
& =1-\operatorname{Pr}\left(\frac{1}{\kappa\left|h_{\mathrm{RR}}\right|^{2}} \geq \gamma_{\mathrm{th}}\right) \operatorname{Pr}\left(\kappa \Psi\left|h_{\mathrm{SR}}\right|^{2}\left|h_{\mathrm{RD}}\right|^{2} \geq \gamma_{\mathrm{th}}\right) \\
& =1-2\left\{1-\exp \left(-\frac{\lambda_{\mathrm{RR}}}{\kappa \gamma_{\mathrm{th}}}\right)\right\} \times \sqrt{\frac{\lambda_{\mathrm{SR}} \lambda_{\mathrm{RD}} \gamma_{\mathrm{th}}}{\kappa \Psi}} \times K_{1}\left(2 \sqrt{\frac{\lambda_{\mathrm{SR}} \lambda_{\mathrm{RD}} \gamma_{\mathrm{th}}}{\kappa \Psi}}\right), \\
P_{4} & =1-\exp \left(-\frac{\lambda_{\mathrm{SD}} \gamma_{\mathrm{th}}}{\Psi}\right) .
\end{aligned}
$$

Finally, substituting (28) and (29) into (27), the OP in this scenario can be expressed as

$$
\begin{aligned}
\mathrm{OP}_{\mathrm{SC}}= & \left\{1-\exp \left(-\frac{\lambda_{\mathrm{SD}} \gamma_{\mathrm{th}}}{\Psi}\right)\right\} \\
& \times\left\{1-2\left\{1-\exp \left(-\frac{\lambda_{\mathrm{RR}}}{\kappa \gamma_{\mathrm{th}}}\right)\right\} \times \sqrt{\frac{\lambda_{\mathrm{SR}} \lambda_{\mathrm{RD}} \gamma_{\mathrm{th}}}{\kappa \Psi}} \times K_{1}\left(2 \sqrt{\frac{\lambda_{\mathrm{SR}} \lambda_{\mathrm{RD}} \gamma_{\mathrm{th}}}{\kappa \Psi}}\right)\right\} .
\end{aligned}
$$




\section{Simulation Results}

The simulation results are given to validate the performance, that is, IP and OP, of our proposed schemes under maximal ratio combining (MRC) and selection combining (SC) techniques. The results are obtained by averaging $10^{5}$ Rayleigh channels [27-29].

In Figures 3 and 4, we investigate the IP and OP as functions of $\Psi(\mathrm{dB})$, where $\gamma_{\mathrm{th}}=1, \alpha=0.5, \eta=1$. One can observe from Figure 3 that as $\Psi$ increases from -5 to $20 \mathrm{~dB}$, the IP performance improves accordingly. The source's transmit power is proportional to $\Psi$ value, since $\Psi$ is defined as a ratio between source transmit power and additive white Gaussian noise. Thus, the higher the $\Psi$ value is, the better the SNR at eavesdropper can be obtained. Furthermore, the IP of the MRC technique outperforms that of the SC technique. It is because the eavesdropper can overhear information from both source $\mathrm{S}$ and relay $\mathrm{R}$ using $\mathrm{MRC}$ while only receiving signals from the source with the SC technique. In Figure 4, the outage performance of MRC is superior to that of the SC method. It is because the destination can combine signals from relay and source in MRC, which only receives this information from relay user in the SC method. As shown from Figures 3 and 4, the performances of both destination $\mathrm{D}$ and eavesdropper $\mathrm{E}$ can be continuously improved by increasing the transmit power. Thus, the designer should select a suitable value of $\Psi$ when designing in practice for trade-off between security and reliability of the system.

Figures 5 and 6 show the IP and OP as a function of $\alpha$ for the time-switching relaying (TSR) protocol, where $\gamma_{\text {th }}=1, \Psi=3 \mathrm{~dB}, \eta=1$. The value of $\alpha$ is crucial, since it influences both the harvested energy at the relay and the information transmission from the relay to the destination. As a result, the higher the value of $\alpha$ is, the more energy the relay can harvest. However, there is less time for information transmission to the destination. Therefore, the OP can obtain the best value at the optimal point of $\alpha$; then the performance worsens. Notably, when the value of $\alpha$ is small, the eavesdropper has a low probability of intercepting the information. For instance, the IPs of MRC and SC are 0.073 and 0.0068 , respectively, when $\alpha$ equals 0.05 . When $\alpha$ is higher than the optimal value, the outage performance and system security are worse. It provides useful information for designing a practical system.

In Figures 7 and 8 , we investigate the IP and OP as a function of rate threshold requirement to decode the signal successfully, where $\alpha=0.85, \Psi=5 \mathrm{~dB}, \eta=1$. As observed from Figures 7 and 8 , as the rate threshold increases from 0.25 to $4 \mathrm{bps} / \mathrm{Hz}$, the IP and OP performance degrades accordingly. It is expected since when the rate requirement is higher, the eavesdropper and destination need to obtain a higher transmission rate to decode the signal. However, the transmission rate is limited by many factors such as channel gain and allocated time for data transmission. One more interesting point is that the IP and OP performances of MRC and SC are converged to a saturation value when the rate threshold increases.

In Figures 9 and 10, we study the influences of energy conversion efficiency on the network performance, that is, IP

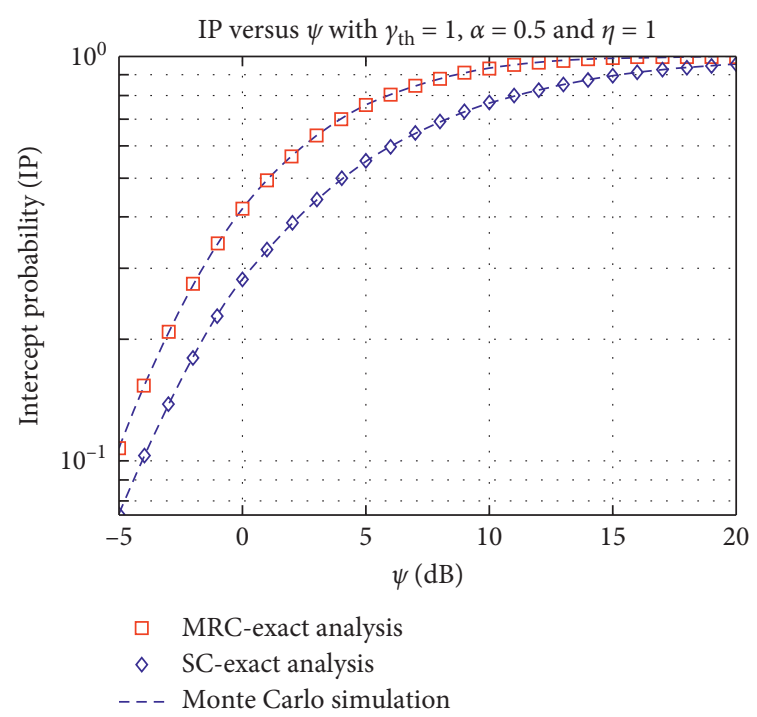

Figure 3: IP versus $\Psi(\mathrm{dB})$.

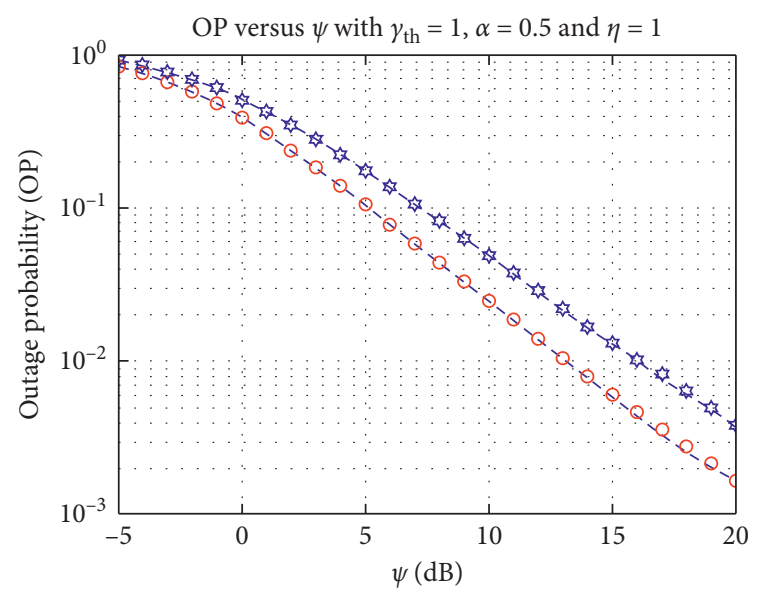

- MRC-exact analysis

$\Leftrightarrow$ SC-exact analysis

- - - Monte Carlo simulation

Figure 4: OP versus $\Psi(\mathrm{dB})$.

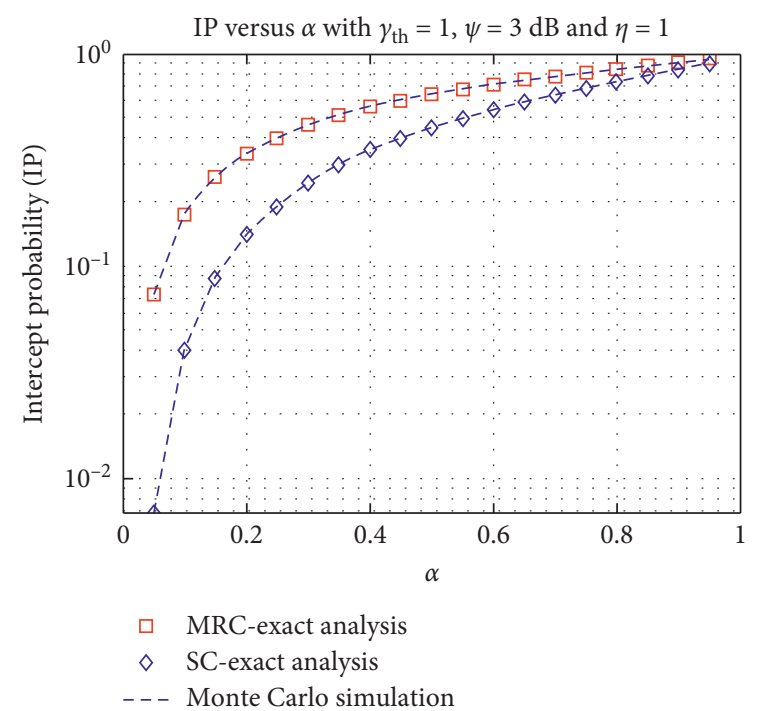

FiguRe 5: IP versus $\alpha$. 


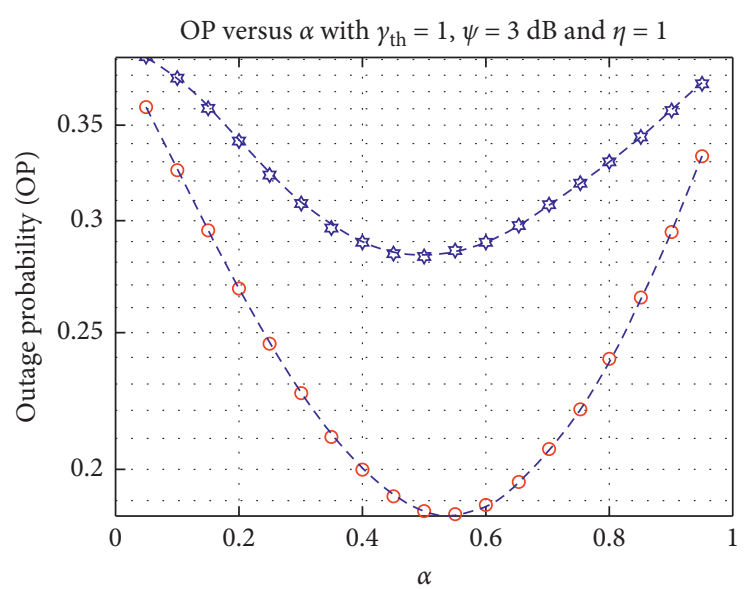

$\begin{array}{cl}\text { - } & \text { MRC-exact analysis } \\ \text { ¿ } & \text { SC-exact analysis } \\ \text { - - } & \text { Monte Carlo simulation }\end{array}$

FIgURE 6: OP versus $\alpha$.

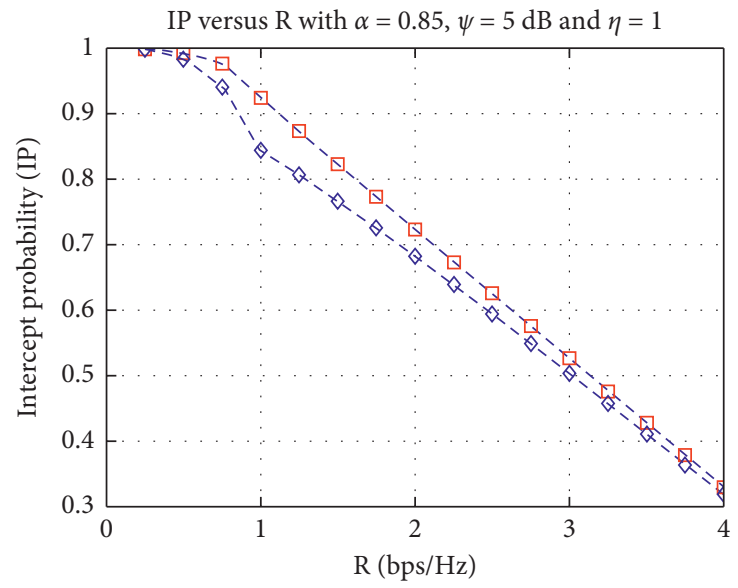

口 MRC-exact analysis

$\diamond \quad$ SC-exact analysis

- - - Monte Carlo simulation

FIgURe 7: IP versus R (bps/Hz).

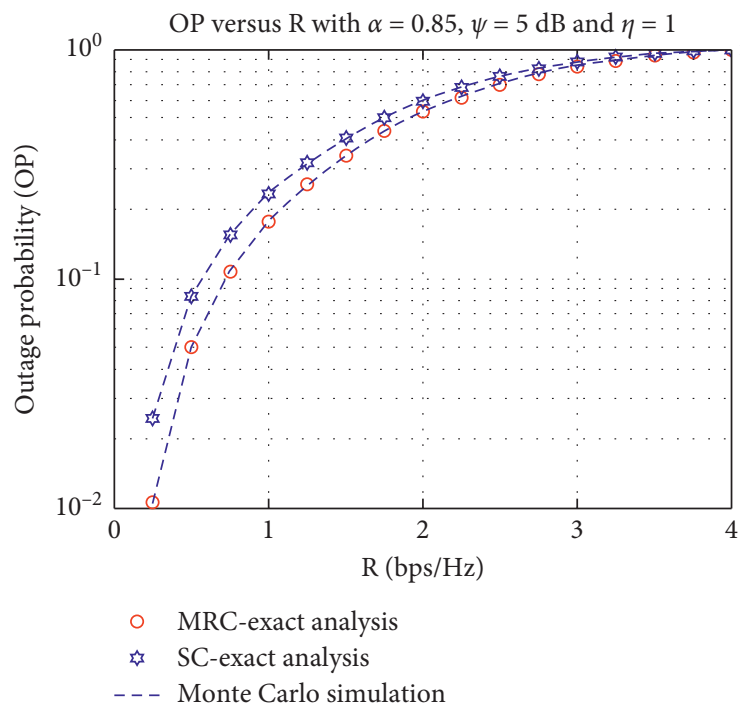

Figure 8: OP versus R (bps/Hz). 


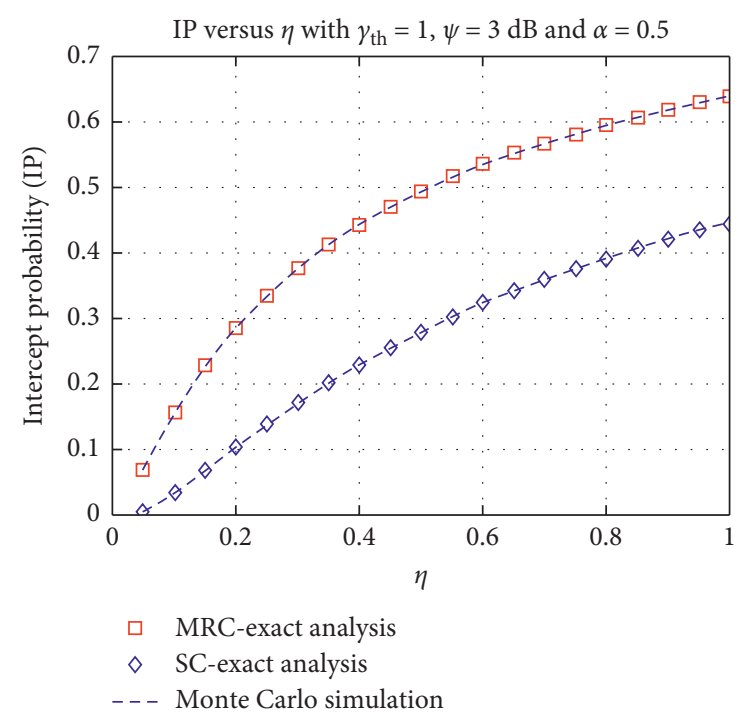

Figure 9: IP versus $\eta$.

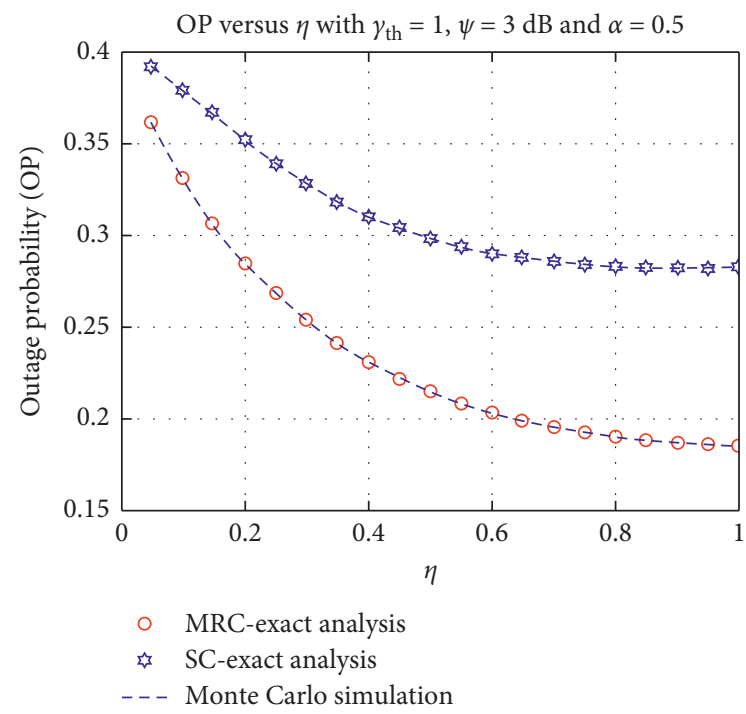

Figure 10: OP versus $\eta$.

and OP. It can be seen that both IP and OP are improved with higher values of IP and OP. This can be explained by the fact that the higher the conversion rate is, the more transmit power at the relay $\mathrm{R}$ can be obtained, which improves the channel gain to eavesdropper and destination. Moreover, similar to Figures 3-8, the IP and OP performance of the MRC is better than that of the SC, which shows the superiority of the MRC technique. However, the MRC technique requires more complicated hardware, which is not always suitable in practice. Thus, the evaluations in our work give different scenarios for the designer to build a system in reality.

\section{Conclusion}

This paper investigated the decode-and-forward (DF) fullduplex (FD) relaying networks under the presence of a direct link. Specifically, the relay node can harvest energy from the source and use it to transmit information to the destination. By considering the above discussions, we derive the closedform expressions of the intercept probability (IP) and the outage probability $(\mathrm{OP})$ in both maximal ratio combining (MRC) and selection combining (SC) techniques at the receiver. Besides, the simulation results show the exactness of the mathematical results compared to simulation ones. Besides, the IP and OP of the MRC technique obtain better performance in comparison to those of the SC technique. In particular, the system security is improved significantly when the time splitting factor value is small. We can extend this work to the case where the source and eavesdropper are equipped with multiple antennas.

\section{Data Availability}

No data were used in this paper. The authors just proposed the system and simulated it by MATLAB.

\section{Conflicts of Interest}

The authors declare that they have no conflicts of interest.

\section{Authors' Contributions}

Phu Tran Tin (phutrantin@iuh.edu.vn) was the main performer, while Dong Si Thien Chau (dongsithienchau@ tdtu.edu.vn), Van-Duc Phan (duc.pv@vlu.edu.vn), Tan N. Nguyen (nguyennhattan@tdtu.edu.vn), and Phu X. Nguyen (phunx4@fpt.edu.vn, phunx4@fe.edu.vn) worked as the advisors of Phu Tran Tin.

\section{Acknowledgments}

This research was supported by the Industrial University of Ho Chi Minh City (IUH), Vietnam, under Grant no. 72/HDDHCN.

\section{References}

[1] L. R. Varshney, "Transporting information and energy simultaneously," in Proceedings of the 2008 IEEE International Symposium on Information Theory, pp. 1612-1616, Toronto, Canada, July 2008.

[2] T. Dinh Hieu, T. T. Duy, and S. G. Choi, "Performance enhancement for harvest-to-transmit cognitive multi-hop networks with best path selection method under presence of eavesdropper," in Proceedings of the 2018 20th International Conference on Advanced Communication Technology (ICACT), pp. 1-2, Chuncheon, Korea (South), February 2018.

[3] Z. Mobini, M. Mohammadi, and C. Tellambura, "Wirelesspowered full-duplex relay and friendly jamming for secure cooperative communications," IEEE Transactions on Information Forensics and Security, vol. 14, no. 3, pp. 621-634, 2019.

[4] T. D. Hieu, G. Sumit, C. Symeon, and O. Bjorn, "Throughput maximization for wireless communication systems with backscatter- and cache-assisted UAV technology," https:// arxiv.org/abs/2011.07955

[5] P. Grover and A. Sahai, "Shannon meets tesla: wireless information and power transfer," in Proceedings of the 2010 
IEEE International Symposium on Information Theory, pp. 2363-2367, Austin, TX, USA, June 2010.

[6] T. N. Nguyen, M. Tran, T.-L. Nguyen, Duy-hung Ha, and Miroslav voznak, "Performance analysis of a user selection protocol in cooperative networks with power splitting protocol-based energy harvesting over nakagami-m/Rayleigh channels," Electronics, vol. 8, pp. 1-14, 2019.

[7] D. H. Tran, T. D. Le, and B. S. Kim, "Stability-aware geographic routing in energy harvesting wireless sensor networks," Sensors, vol. 16, pp. 1-15, 2016.

[8] T. N. Nguyen, T. H. Quang Minh, T. Phuong, P. T. Tran et al., "Performance enhancement for energy harvesting based twoway relay protocols in wireless ad-hoc networks with partial and full relay selection methods," Ad Hoc Networks, vol. 84, pp. 178-187, 2019.

[9] D.-H. Tran, T. X. Vu, S. Chatzinotas, S. ShahbazPanahi, and B. Ottersten, "Coarse trajectory design for energy minimization in UAV-enabled," IEEE Transactions on Vehicular Technology, vol. 69, no. 9, pp. 9483-9496, 2020.

[10] T. N. Nguyen, T. Minh, P. T. Tran, and M. Voznak, “Adaptive energy harvesting relaying protocol for two-way half duplex system network over rician fading channels," Wireless Communications and Mobile Computing, vol. 2018, Article ID 7693016, 10 pages, 2018.

[11] R. Zhang and C. K. Ho, "MIMO broadcasting for simultaneous wireless information and power transfer," IEEE Transaction on Wireless Communications, vol. 12, no. 5, pp. 1989-2001, 2013.

[12] L. Dai, B. Wang, M. Peng, and S. Chen, "Hybrid precodingbased millimeter-wave massive mimo-noma with simultaneous wireless information and power transfer," IEEE Journal on Selected Areas in Communications, vol. 37, no. 1, pp. 131-141, 2019.

[13] T. N. Nguyen, P. T. Tran, and M. Voznak, "Wireless energy harvesting meets receiver diversity: a successful approach for two-way half-duplex relay networks over block Rayleigh fading channel," Computer Networks, vol. 172, Article ID 107176, 2020.

[14] A. Prathima, D. S. Gurjar, H. H. Nguyen, and A. Bhardwaj, "Performance analysis and optimization of bidirectional overlay cognitive radio networks with hybrid-SWIPT," IEEE Transactions on Vehicular Technology, vol. 69, no. 11, pp. 13467-13481, 2020.

[15] Y. Liu, Y. Ye, H. Ding, F. Gao, and H. Yang, "Outage performance analysis for SWIPT-based incremental cooperative NOMA networks with non-linear harvester," IEEE Communications Letters, vol. 24, no. 2, pp. 287-291, 2020.

[16] D. S. Gurjar, H. H. Nguyen, and P. Pattanayak, "Performance of wireless powered cognitive radio sensor networks with nonlinear energy harvester," IEEE Sensors Letters, vol. 3, no. 8 , pp. 1-4, Article ID 7500704, 2019.

[17] D.-H. Ha, T. N. Nguyen, M. H. Q. Tran, X. Li, P. T. Tran, and M. Voznak, "Security and reliability analysis of a two-way half-duplex wireless relaying network using partial relay selection and hybrid TPSR energy harvesting at relay nodes," IEEE Access, vol. 8, pp. 187165-187181, 2020.

[18] Q. V. Do, T.-N.-K. Hoan, and I. Koo, "Optimal power allocation for energy-efficient data transmission against fullduplex active eavesdroppers in wireless sensor networks," IEEE Sensors Journal, vol. 19, no. 13, pp. 5333-5346, 2019.

[19] P. T. Tin, B. H. Dinh, T. N. Nguyen, D. H. Ha, and T. T. Trang, "Power beacon-assisted energy harvesting wireless physical layer cooperative relaying networks: performance analysis," Symmetry, vol. 12, no. 1, 2020.
[20] A. Arafa, W. Shin, M. Vaezi, and H. V. Poor, "Secure relaying in non-orthogonal multiple access: trusted and untrusted scenarios," IEEE Transactions on Information Forensics and Security, vol. 15, pp. 210-222, 2020.

[21] Tin Phu Tran, Dang The Hung, N. Tan, D. Tran, and M. Voznak, "Secrecy performance enhancement for underlay cognitive radio networks employing cooperative multi-hop transmission with and without presence of hardware impairments," Entropy, vol. 21, no. 2, pp. 1-16, 2019.

[22] L. Xingwang, Z. Mengle, L. Yuanwei, L. Lihua, D. Zhiguo, and N. Arumugam, "Secrecy analysis of ambient backscatter NOMA systems under I/Q imbalance," IEEE Transactions on Vehicular Technology, vol. 69, no. 10, pp. 12286-12290, 2020.

[23] H. Dinh Tran, D. Trung Tran, and S. G. Choi, "Secrecy performance of a generalized partial relay selection protocol in underlay cognitive networks," International Journal of Communication Systems, vol. 31, no. 17, Article ID e3806, 2018.

[24] T. N. Nguyen, M. Tran, D. H. Ha, T. T. Trang, and M. Voznak, "Multi-source in DF cooperative network with PRS protocol based full-duplex energy harvesting over a Rayleigh fading channel: performance analysis," Proceedings of the Estonian Academy of Sciences, vol. 68, no. 3, 2019.

[25] D. H. Tran, V. D. Nguyen, G. Sumit, C. Symeon, X. T. Vu, and O. Bjorn, "UAV relay-assisted emergency communications in IoT networks: resource allocation and trajectory optimization," https://arxiv.org/abs/2008.00218.

[26] D. Zwillinger, Table of Integrals, Series, and Products, Academic Press, Springer, New York, NY, USA, 2015.

[27] T. Nguyen, T. Quang Minh, P. Tran, and M. Vozňák, "Energy harvesting over rician fading channel: a performance analysis for half-duplex idirectional sensor networks under hardware impairments," Sensors, vol. 18, no. 6, 2018.

[28] P. Tran Tin, T. N. Nguyen, M. Tran, and L. Sevcik, "Exploiting direct link in two-way half-duplex sensor network over block Rayleigh fading channel: upper bound ergodic capacity and exact SER analysis," Sensors, vol. 20, no. 4, 1165 pages, 2020.

[29] D. Le, D. H. Tran, S. Choi, B. Kim, and B. An, "Impact of beamforming on the path connectivity in cognitive radio adhoc networks," Sensors, MDPI, vol. 17, no. 4, 2016. 